\title{
THE COMPRESSION OF YIELDING LOOPS
}

I. M. STUART and R. K. MANN

(Received 4 April 1967; revised 28 September 1967)

\section{Introduction}

In studying the compression of loops of fabric between flat parallel plates, the problem arises of the compression of bends. A 'bend' is illustrated in Figs. 1 and 2. Two bends are joined to form a loop. The material is postu-

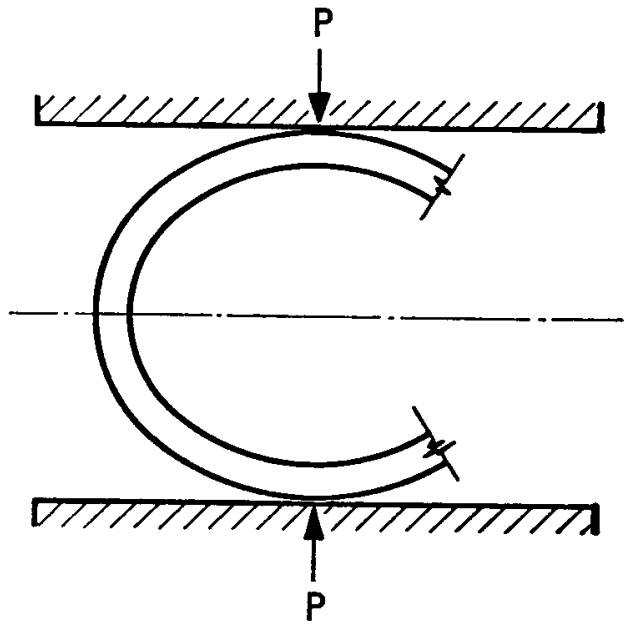

Fig. 1. The loading of a bend.

lated to yield in bending such that the bending moment

$$
M=B\left(K_{I}-K_{R}\right)
$$

where $B$ is a bending rigidity, $K_{I}$ the current impressed curvature, and $K_{R}$ the 'remanent' curvature. This remanent curvature is the free, or natural curvature in the material caused by its past and present deformation. We study the case when the remanent curvature

$$
K_{R}=\lambda \max K_{I}
$$

that is, the remanent curvature is proportional to the greatest previously impressed curvature. We call equations (1) and (2) the 'Remanent Curvature Hypothesis'. 
Stuart and Mann have shown (6) that such a relationship between remanent curvature and impressed curvature is a reasonable extension of the notion of 'recovery angle' after a material has been bent to form a half circle shape. This is a standard test of recovery from creasing (2), (3), for woven cloth.

Waters (4) and Stechert (5) have studied the case of a 'bend' of an elastica. A more complete study of the differential equation governing the bend shape is given by Stuart et al. (1), when the relationship between bending moment and curvature is not prescribed, and when the bend can have a non-zero curvature where it contacts the compressing plates.

We proceed to analyse this more general case when the bending is described by the Remanent Curvature Hypothesis. We consider both the loading and unloading of the bend, and give the results of some calculations with some comparison with experimental results of the loading and unloading of loop of a wool worsted cloth.

\section{Analysis}

The loading of a loop between parallel plates is somewhat akin to the bending of a length of yielding wire between the jaws of a pair of pliers (see Fig. 3). The piece of wire between the jaws is gradually diminishing

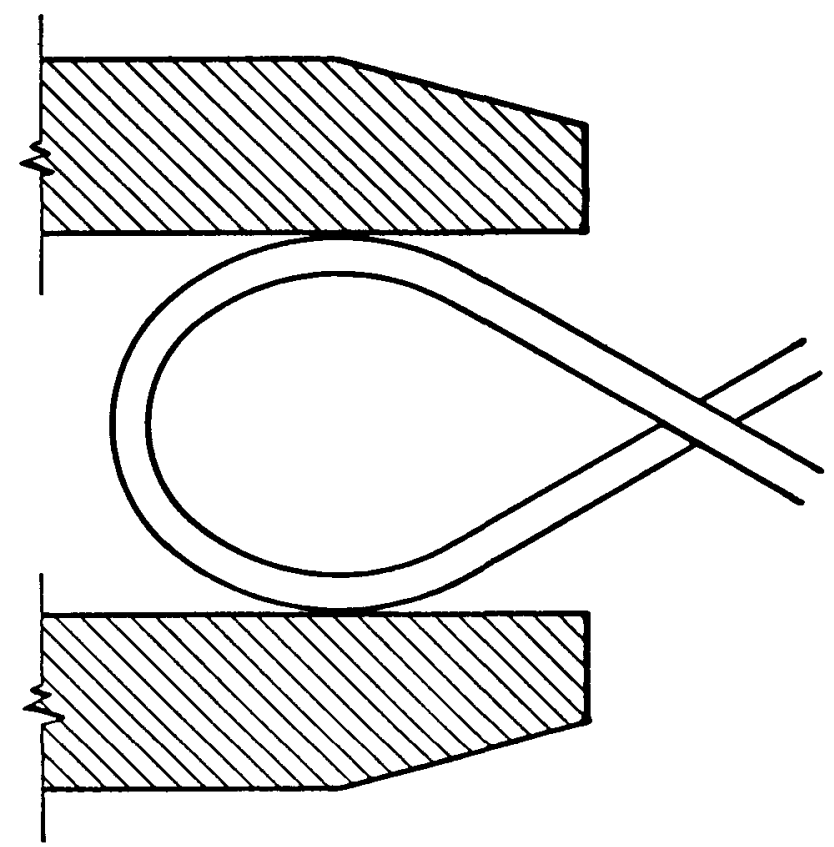

Fig. 3. Wire being bent between the jaws of a pair of pliers. 


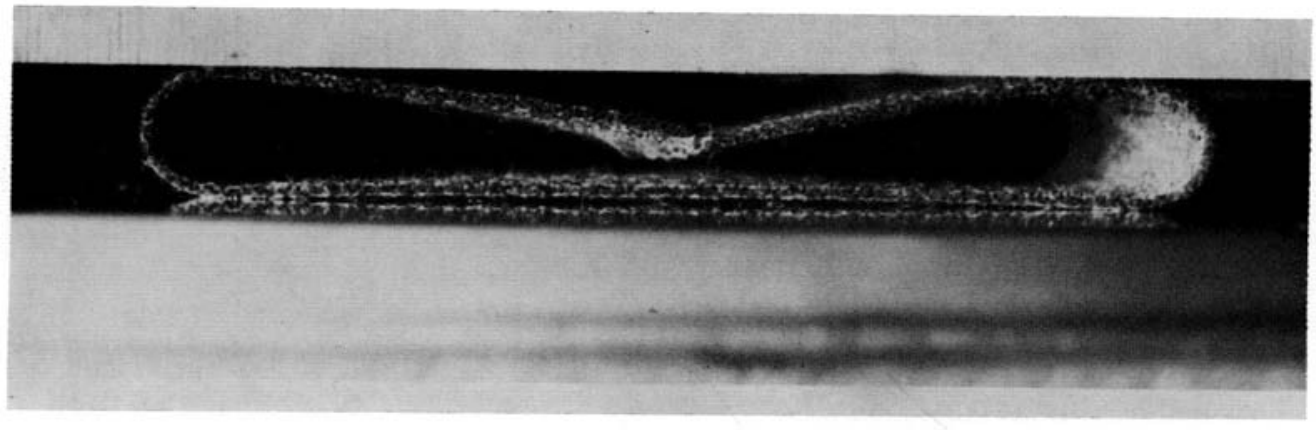

(a)

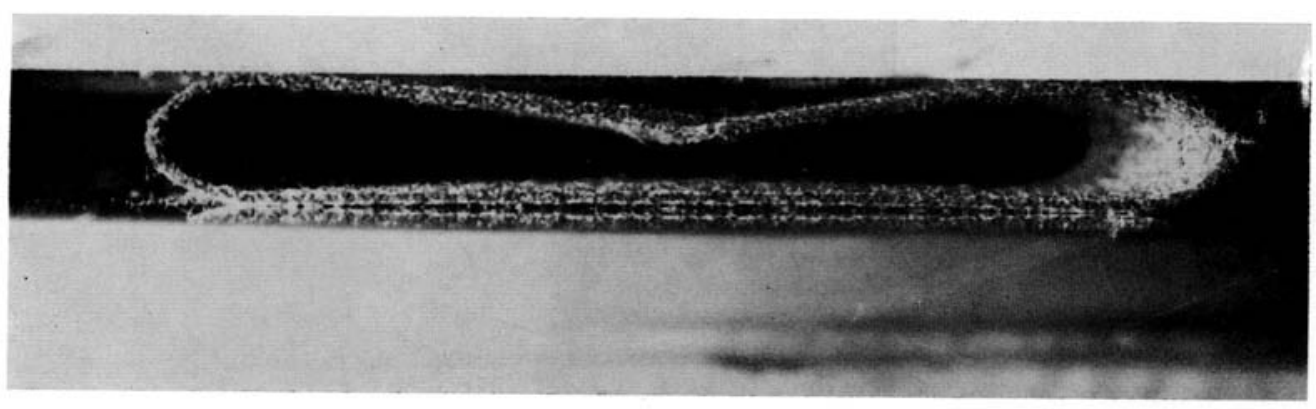

(b)

Fig. 2. Photographs of a loop of cloth (a) loading (b) unloading. 
so that the place on the wire in contact with the jaws has been previously between the jaws, and suffered some deformation and possibly has yielded prior to being in contact with the jaws. If there is no moment being applied to the wire in contact with the jaws as is the case under assumption A below, the curvature there is that due to the past history of its deformation between the jaws.

The flexural properties of the loop are assumed to be constant so that the bend is symmetrical about a plane half-way between the plates. We describe the bend by the distance $s$ along it from this plane of symmetry, and the angle $\theta$ made by the bend with this plane. The senses taken by the shear force $N$, the tension $T$ and the bending moment $M$ are indicated in Figure 4(a).

It follows from symmetry and equilibrium of the bend between $\theta=0$ and $\theta=\pi$ that the tensions at $\theta=0$ and $\theta=\pi$ are zero.

The equilibrium of the segment of bend $(\theta, 0)$ shown in Figure $4(\mathrm{~b})$ requires that

$$
N=-P \cos \theta
$$

where $P$ is the load supported by the bend.

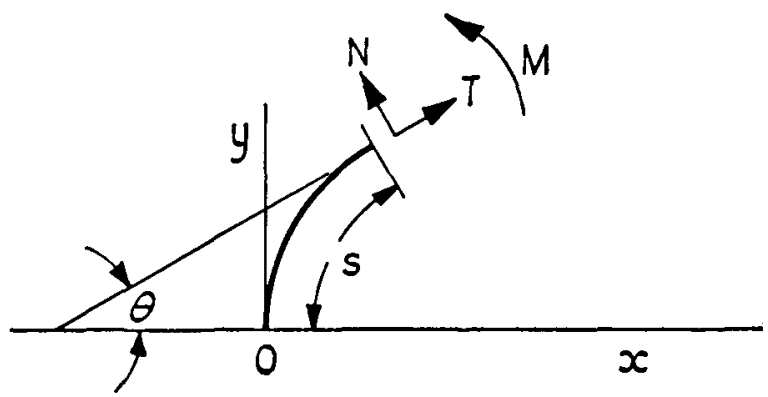

(a)

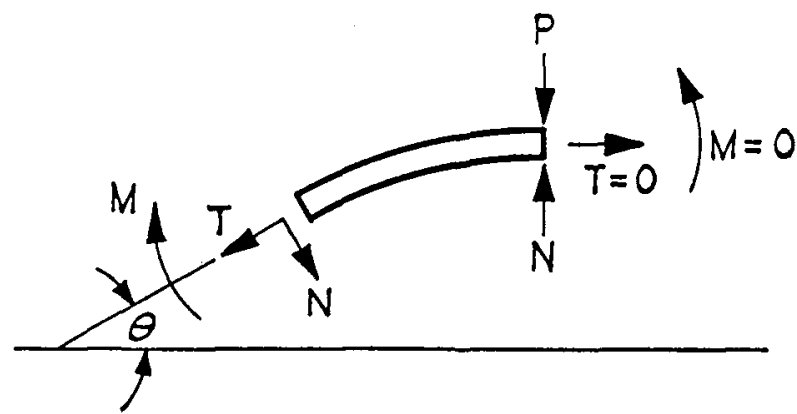

(b)

Fig. 4. (a) Senses of $N, T, M$ acting at point $(s, \theta)$ on a bend. (b) Forces and moments acting on the bend on the interval $(\theta, 0)$, under assumption $A$. 
It follows from the equilibrium equations of elementary bending theory that

$$
\frac{d M}{d s}=-N
$$

So the differential equation governing the bend shape which applies irrespective of bending and yielding properties of the bend material is

$$
\frac{d M}{d s}=P \cos \theta
$$

\section{Boundary Conditions}

We consider two sets of boundary conditions. The first set - which we call assumption $\mathrm{A}$ - follows when we assume that the material outside the bend has no influence on the material inside the bend. The boundary conditions then become:

at $s=0, \theta=\frac{1}{2} \pi$, (by symmetry and continuity), and

when $\theta=0, \pi ; T=0$, and $M=0$.

The second set of boundary conditions, assumption B, corresponds to the situation where the material between the compressing plates is held flat against the plates by a moment due to interference between the parts of the loop outside the bends. The condition of zero moment at $\theta=0$ and $\pi$ is replaced by the condition that the curvature is zero at these points.

\subsubsection{Analysis of the loading of a bend under assumption $A$}

When the bending moment $M$ is related to the current impressed curvature,

$$
K_{I}=\frac{d \theta}{d s},
$$

and to the remanent curvature $K_{R}$ by equation (1), then equation (3) becomes

$$
B \frac{d}{d s}\left(\frac{d \theta}{d s}-K_{R}\right)=P \cos \theta .
$$

The boundary conditions are:

$$
\begin{aligned}
& s=0, \theta=\frac{1}{2} \pi ; \\
& \theta=0, \pi ; \text { from } M=0, \frac{d \theta}{d s}=K_{R}
\end{aligned}
$$

and the remanent curvature $K_{R}$ is given by equation (2). 
We put

$$
c^{2}=\frac{B(1-\lambda)}{P}
$$

and

$$
\boldsymbol{\sigma}=s / c
$$

Then equation (4) becomes

$$
\frac{d}{d \sigma}\left(\frac{d \theta}{d \sigma}-c K_{R}\right)=(1-\lambda) \cos \theta
$$

For load increasing we denote by the suffix * parameters associated with the greatest load yet reached. Thus $P_{*}$ is the greatest load yet applied, and

$$
c_{*}^{2}=\frac{B(1-\lambda)}{P_{*}}
$$

In equation (6) the only load dependent term is

$$
\frac{d}{d \sigma}\left(c K_{R}\right)
$$

Now

$$
c K_{R}=c\left(\frac{d \theta}{d s}\right)_{R}=\left(\frac{d \theta}{d \sigma}\right)_{R}=k_{R}
$$

say, the remanent curvature expressed in dimensionless terms. We now suppose that $k_{R}$ is independent of $c$. This assumption is equivalent to assuming that the forms of the bend at different loads are similar, and reducible to the same shape by a scale factor.

Now, let the solution of equation (6) with boundary conditions stated be given by

$$
\frac{d \theta}{d \sigma}=f(\sigma, \lambda)
$$

That is

$$
c K(s)=c\left(\frac{d \theta}{d s}\right)=f\left(\frac{s}{c}, \lambda\right) .
$$

(Note: As $\theta$ decreases from $\frac{1}{2} \pi$ to $0, d \theta / d \sigma$ is negative.)

In the context of increasing load on the bend the remanent curvature hypothesis, given by equation (2) can be written

$$
\left|K_{* R}(s)\right|=\lambda \underset{c \geqq c_{*}}{\operatorname{Max}}\left|K_{I}(s)\right| .
$$

Now equation (9) gives the curvature at $s$ for all values of $c$. Differentiating equation (9) with respect to $c$ keeping $s$ fixed 


$$
K(s)+c \frac{\partial K(s)}{\partial c}=\left(\frac{-s}{c^{2}}\right) \frac{\partial f(s / c, \lambda)}{\partial(s / c)} .
$$

We put $(\partial K(s) / \partial c)=0$ to obtain the condition for $K(s)$ stationary, and substitute for $K(s)$ using equation (9) to obtain

$$
f\left(\frac{s}{c}, \lambda\right)+\frac{s}{c} \frac{\partial f(s / c, \lambda)}{\partial(s / c)}=0
$$

or

$$
\frac{\partial}{\partial(s / c)}\left\{\frac{s}{c} \partial f\left(\frac{s}{c}, \lambda\right)\right\}=0
$$

or

$$
\frac{\partial}{\partial \sigma}\{\sigma t(\sigma, \lambda)\}=0
$$

We find by calculation that this stationary value is in fact a maximum of the absolute value of the curvature $|K(s)|$.

Let this equality of equation (11) occur when $\sigma=\sigma_{M}$. For a particular distance $s$ along the bend, this value $\sigma_{M}$ of $\sigma$ prescribes a value of $c, c_{M}$ say, which indicates a value of the load applied when the value of the curvature imposed at $s$ has been stationary. $c_{M}$ and $\sigma_{M}$ are related by

$$
\sigma_{M}=\frac{s}{c_{M}}
$$

For values of $c_{M}>c_{*}$ (or $\left.\sigma_{*}>\sigma_{M}\right)$, the stationary value of the curvature has been achieved at a load less than the current load.

By equation (9) the value of this curvature is given by

$$
c_{M} K_{M}(s)=f\left(\sigma_{M}, \lambda\right)
$$

or substituting $s / \sigma_{M}$ for $c_{M}$,

$$
K_{M}(s)=\frac{\sigma_{M} f\left(\sigma_{M}, \lambda\right)}{s}
$$

So, for $\sigma_{*}>\sigma_{M}$ the remanent curvature expressed in dimensionless terms

$$
k_{R}=c_{*} K_{R}(s)=\frac{\lambda\left[\sigma_{M} f\left(\sigma_{M}, \lambda\right)\right]}{\sigma_{*}} .
$$

On the other hand, for $c_{M}<c_{*}$ the current curvature is the greatest yet applied.

So, for $\sigma_{*}<\sigma_{M}$,

$$
k_{R}=\lambda\left(\frac{d \theta}{d \sigma_{*}}\right)
$$


Thus the differential equations to be integrated are as follows:

$$
\frac{d}{d \sigma}\left(\frac{d \theta}{d \sigma}-k_{R}\right)=(1-\lambda) \cos \theta
$$

where $k_{R}=\lambda(d \theta / d \sigma)$ for $\sigma<\sigma_{M}$ and

$$
k_{R}=\frac{\lambda}{\sigma}[\sigma f(\sigma, \lambda)]_{M} \text { for } \sigma \geqq \sigma_{M}
$$

where $[\sigma f(\sigma, \lambda)]_{M}$ is the stationary value of $\sigma(d \theta / d \sigma)$ occurring when $\sigma=\sigma_{M}$.

The boundary conditions are:

$$
\begin{aligned}
& \sigma=0, \quad \theta=\frac{1}{2} \pi, \\
& \theta=0, \quad \frac{d \theta}{d \sigma}=\frac{\lambda}{\sigma}[\sigma f(\sigma, \lambda)]_{M},
\end{aligned}
$$

or

$$
\frac{d}{d \sigma}\left\{\theta-\lambda[\sigma f(\sigma, \lambda)]_{M} \log \sigma\right\}=0
$$

The procedure for solution of equation (12) is thus as follows.

To start the integration it is necessary to estimate a value of $(d \theta / d \sigma)_{\sigma=0}$, say $a_{1}$. Equation (6) is then integrated from $\sigma=0$, starting with $\theta=\frac{1}{2} \pi$, $(d \theta / d \sigma)=a_{1}$ and $k_{R}=\lambda(d \theta / d \sigma)$.

Thus we have:

$$
\frac{d}{d \sigma}\left(\frac{d \theta}{d \sigma}-\lambda \frac{d \theta}{d \sigma}\right)=(1-\lambda) \cos \theta
$$

or $\left(d^{2} \theta / d \sigma^{2}\right)=\cos \theta$.

We proceed with this integration until $\sigma(d \theta / d \sigma)$ has a stationary value, $\sigma_{M_{1}}(d \theta / d \sigma)_{M_{1}}$ say, with the value of $\sigma, \sigma_{M_{1}}$.

We then change to the differential equation

$$
\frac{d}{d \sigma}\left(\frac{d \theta}{d \sigma}-\frac{\lambda}{\sigma} \sigma_{M_{1}}\left(\frac{d \theta}{d \sigma}\right)_{M_{1}}\right)=(1-\lambda) \cos \theta,
$$

and integrate until

$$
\theta-\lambda \sigma_{M_{1}}\left(\frac{d \theta}{d \sigma}\right)_{M_{1}} \log \sigma
$$

has a stationary value.

At this value of $\sigma, \sigma_{01}$ say, $\theta$ should be zero. If it is not, we take a second value of $(d \theta / d \sigma)_{\sigma=0}, a_{2}$ say, and repeat the integration, repeating until $\theta=0$ at the stationary point. 
AbBreviated PROCEDURE. Starting at $\sigma=0$ with $\theta=\frac{1}{2} \pi,(d \theta / d \sigma)=a_{1}$ (which will be negative), integrate the equation:

$$
\frac{d^{2} \theta}{d \sigma^{2}}=\cos \theta
$$

inspecting $\sigma(d \theta / d \sigma)=\beta(\sigma)$ say.

When $\sigma(d \theta / d \sigma)$ turns up, find $\sigma_{M_{1}}, \beta_{M_{1}}$ at the stationary point.

Then change equation to

$$
\frac{d^{2} \theta}{d \sigma^{2}}=(1-\lambda) \cos \theta-\frac{\lambda \beta_{M_{1}}}{\sigma^{2}}
$$

and integrate until

$$
\theta-\lambda \beta_{M_{1}} \log \sigma
$$

reverses direction, when we find the values $\sigma_{01}, \theta\left(\sigma_{01}\right)$ at the stationary point.

If $\theta\left(\sigma_{01}\right) \neq 0$, we select a new value of $a, a_{2}$, and repeat. Thus we integrate $j$ times until $\theta\left(\sigma_{0 j}\right)=0$ (to a sufficient limit of accuracy).

\subsubsection{Analysis of the unloading of a bend under assumption $A$}

We consider the shape of the bend as the load is decreased after having been applied to a maximum value of $P_{*}$.

The remanent curvature will be as follows:

$$
\begin{aligned}
K_{R} & =\lambda \cdot K\left(c_{*}\right) & \text { for } s<c_{*} \sigma_{M} \\
& =\frac{\lambda \beta_{m}}{s} & \text { for } c_{*} \sigma_{M}<s
\end{aligned}
$$

which corresponds to the remanent curvature being determined by the maximum curvature during loading.

The differential equation is then:

$$
B \frac{d}{d s}\left(\frac{d \theta}{d s}-K_{R}\right)=P \cos \theta
$$

and putting

we obtain

$$
\frac{B(1-\lambda)}{P_{*}}=c_{*}^{2}, \quad \frac{B(1-\lambda)}{P}=c^{2}, \quad \sigma=\frac{s}{c_{*}}
$$

$$
\frac{d}{d \sigma}\left(\frac{d \theta}{d \sigma}-c_{*} K_{R}\right)=\left(\frac{c_{*}}{c}\right)^{2}(1-\lambda) \cos \theta
$$

with $c_{*} K_{R}$ given by: 
(a) for $0<\sigma<\sigma_{m}(\lambda)$

$$
K_{R}=\lambda K_{* I}
$$

or

(b) for $\sigma>\sigma_{m}$

$$
c_{*} K_{R}=\lambda\left(\frac{d \theta}{d \sigma}\right)_{c=c_{*}}
$$

$$
K_{R}=\frac{\lambda \beta_{m}(\lambda)}{s}
$$

or

$$
c_{*} K_{R}=\frac{\lambda \beta_{m}(\lambda)}{\sigma}
$$

The relevant equations become:

$$
\begin{aligned}
& \frac{d^{2} \theta}{d \sigma^{2}}=\left(\frac{c_{*}}{c}\right)^{2}(1-\lambda) \cos \theta+\lambda\left(\frac{d^{2} \theta}{d \sigma^{2}}\right)_{c=c_{*}} \\
& \frac{d^{2} \theta}{d \sigma^{2}}=\left(\frac{c_{*}}{c}\right)^{2}(1-\lambda) \cos \theta-\lambda \frac{\beta_{m}(\lambda)}{\sigma^{2}} .
\end{aligned}
$$

\subsection{Assumption B}

The end condition of zero moment at $\theta=0$ can be replaced by the assumption (B) that the curvature is zero at that point, corresponding to the situation where the material between the compressing plates is held flat against the plates by a moment due to interference between the parts of the loop outside the bend. This is probably a closer approximation to the conditions of our tests than the assumption (A) of no interference.

\subsubsection{Loading of the Bend under Assumption B}

Because of the straightening moment at its end, part of the loop, in bending, will reach a curvature less than a previous remanent curvature $\lambda \beta m / s$, and so we assume its remanent curvature to be reduced to

$$
K_{R}=\frac{\lambda \beta_{m}}{s}-\lambda_{R}\left(\frac{\lambda \beta_{m}}{s}-\frac{d \theta}{d \sigma}\right)
$$

or

$$
c K_{R}=\left(1-\lambda_{R}\right) \frac{\lambda \beta_{m}}{\sigma}+\lambda_{R} \frac{d \theta}{d \sigma}
$$

where $0 \leqq \lambda_{R}<1$ and $\lambda_{R}$ is not necessarily equal to $\lambda$. This equation applies for $s>s_{R}$ where, at $s=s_{R},(d \theta / d s)=\left(\lambda \beta_{m} / s\right)$ which reduces to the same condition as the endpoint under the previous assumption; that is, $\sigma(d \theta / d \sigma)$ 
has a minimum. The endpoint to be found under assumption $B$ is when $\theta$ has a minimum $(d \theta / d \sigma=0)$, where $\theta$ should also be zero. For $s<s_{R}$, the equations are as for the previous assumption.

\subsubsection{Unloading of the Bend under Assumption B}

As the bend is unloaded, some of the material which had had its remanent curvature reduced during bending may again experience an increase in that curvature. This is assumed to happen when:

$$
-\lambda \frac{d \theta}{d s}>-K_{R}
$$

and thus the remanent curvature in unloading is given by:

(a) $0<s<c_{*} \sigma_{m}: c_{*} K_{R}=\lambda\left(\frac{d \theta}{d \sigma}\right)_{c_{*}=\mathrm{c}}$
(b) $c_{*} \sigma_{m}<s<c_{*} \sigma_{R}: c_{*} K_{R}=\frac{\lambda \beta_{m}(\lambda)}{\dot{\sigma}}$

(c) $c_{*} \sigma_{R}<s<c_{*} \sigma_{0}: c_{*} K_{R}=-\max \left\{\begin{array}{l}-\left[\lambda\left(1-\lambda_{R}\right) \frac{\beta m}{\sigma}+\lambda_{R}\left(\frac{d \theta}{d \sigma}\right)_{c_{*}=c}\right. \\ -\lambda \frac{d \theta}{d \sigma}\end{array}\right.$

(d) $s>c_{*} \sigma_{0}: c_{*} K_{R}=-\max \left\{\begin{array}{l}-\lambda\left(1-\lambda_{R}\right) \frac{\beta m}{\sigma} \\ -\lambda \frac{d \theta}{d \sigma}\end{array}\right.$

\section{Method of Solution of the Equations}

The equations describing the loop were solved by numerical integration on the CDC3600 computer of the CSIRO Scientific Computing Network.

Each equation was reduced to the form:

$$
\frac{d \theta}{d \sigma}=G\left(\frac{c_{*}}{c}, \lambda\right) \cos \theta+Q(\sigma)
$$

and the three point approximation for $d^{2} \theta / d \sigma^{2}$ was used:

$$
\left(\frac{d^{2} \theta}{d \sigma^{2}}\right)_{0}=\frac{\theta_{-1}-2 \theta_{0}+\theta_{+1}}{H^{2}}
$$

where $H$ is the interval size in $\sigma$ to be used for the step by step integration. 
At $\sigma=0, \theta=\frac{1}{2} \pi$ and $d \theta / d \sigma=-a$. An approximation obtained by a rough analysis was $a \cong \sqrt{ } \mathbf{2}$ which was used as a first estimate, $a_{1}$.

Thus an array, $\theta(n)$, where $n$ is the step number, was built up as in Table I. $(n=\sigma / H+1$.

TABLE I

\begin{tabular}{|c|c|c|}
\hline$n$ & $\sigma$ & $\theta n$ \\
\hline 1 & 0 & $\sigma_{1}=\frac{1}{2} \pi$ \\
\hline 2 & $H$ & $\theta_{2}=\frac{1}{2} \pi-a H$ \\
\hline 3 & $2 H$ & $\theta_{3}=2 \theta_{2}-\theta_{1}+H^{2} \cdot Q(H)$ \\
\hline 4 & $3 H$ & $\begin{array}{l}\theta_{1}=2 \theta_{3}-\theta_{2}+H^{2} K \cos \theta_{2}+H^{2} \cdot Q(2 H) \\
\text { etc. }\end{array}$ \\
\hline
\end{tabular}

The bending situation of the case under consideration was first solved as indicated in Table III. $c_{*} \sigma_{0}$ is the length of the half loop, and $\theta_{0}$ which should be zero, is the error associated with $a_{1}$.

If $\theta_{0}$ was outside acceptable limits (usually \pm 0.0001 ) a correction was made to ' $a$ ' by:

$$
a_{2}=a_{1}\left(1+m \theta_{0}\right)
$$

where, empirically, $m=0.5$ was found suitable. The integration, using ' $a_{2}$ ', was repeated. Further corrections to ' $a$ ' were by linear, and then quadratic, interpolation, based on the values of $\theta_{\mathbf{0}}$.

TABLE II

\begin{tabular}{lll}
\hline$R_{n}$ & Assumption A & Assumption B \\
\hline $2 a H$ & $\begin{array}{c}n=1 \\
2<n<n_{n}\end{array}$ \\
$\theta_{n+1}-\theta_{n-1}$ & $n>n_{m}$ & $n_{m}<n<n_{R}$ \\
$\frac{2 \beta m}{n-1}$ & - & $n_{R}<n<n_{0}$ \\
$\frac{2 \beta m(1-\lambda)}{n-1}+\frac{\lambda_{R}}{\lambda}\left(\theta_{n+1}-\theta_{n-1}\right)$ & - & $n>n_{0}$ \\
$\frac{2 \beta m(1-\lambda)}{n-1}$ & - where $n_{m}=\frac{\sigma m}{H}+1$, etc.
\end{tabular}


Table III

\begin{tabular}{llll}
\hline Integrate with & $\begin{array}{l}\text { Inspecting for } \\
\text { minimum of }\end{array}$ & $\begin{array}{l}\text { Determine at the } \\
\text { minimum }\end{array}$ \\
\hline$G$ & $Q$ &
\end{tabular}

Assumption A, Bending
(1) 1
0
$\sigma \frac{d \theta}{d \sigma}$
$\sigma m, \beta m=\left(\sigma \frac{d \theta}{d \sigma}\right)_{M}$
(2) $(1-\lambda)$
$\frac{\lambda \beta m}{\sigma^{2}}$
$\theta+\lambda \beta m \log \sigma$
$\sigma_{0}, \theta_{0}$

Assumption $A$, Unbending

$$
\left(\frac{c_{*}}{c}\right)^{2}(1-\lambda) \quad \frac{\lambda}{4 H^{2}}\left(R_{n+1}-R_{n-2}\right) \quad \theta+\lambda \beta m \log \sigma \quad \sigma_{0}, \theta_{0}
$$

Assumption B, Bending
(1) 1
0
$\sigma \frac{d \theta}{d \sigma}$
$\sigma m, \beta m$
(2) $(1-\lambda)$
$\frac{\lambda \beta m}{\sigma^{2}}$
$\theta+\lambda \beta m \log \sigma$
$\sigma R$
(3) $\frac{1-\lambda}{1-\lambda_{R}}$
$\frac{\lambda \beta m}{\sigma^{2}}$
$\theta$
$\sigma_{0}, \theta_{0}$

Assumption $B$, Unbending
(1) $\left(\frac{c_{*}}{c}\right)^{2}(1-\lambda)$
$\frac{\lambda}{4 H^{2}}\left(R_{n+1}-R_{n-1}\right)$
$\theta$
$\sigma_{0}, \theta_{0}$

Also testing for $\left(\theta_{n+1}-\theta_{n-1}\right)>\left(-R_{n}\right)$ in which case change to (2):
(2) $\left(\frac{c_{*}}{c}\right)^{2}$
0
testing for $\left(\theta_{n-1}-\theta_{n+1}\right)<\left(-R_{n}\right)$ when revert to (1). After solution, replace $R_{n}$ in table by $R_{n}=\theta_{n+1}-\theta_{n-1}$, where (2) was used. of:

Once the bending case was solved, an array was filled giving the values

$$
R_{n}=\frac{2 H}{\lambda}\left(c_{*} K_{R}\right)
$$

a modified form of the remanent curvature, as in Table II. This was used to solve the unbending case, $c^{*} / c<1$ with successive values of $\left(c^{*} / c\right)$ decreasing from 1.0, usually $1(0.02) 0.10$. The value of $a_{1}$ was found by extrapolation from values found for previous values of $c_{*} / c$. Solutions have been obtained for a variety of values of $\lambda$ (and $\lambda_{R}$ ), and the arrays $\theta(n)$ were used to find parameters of the loops.

The main output of the program was either: 

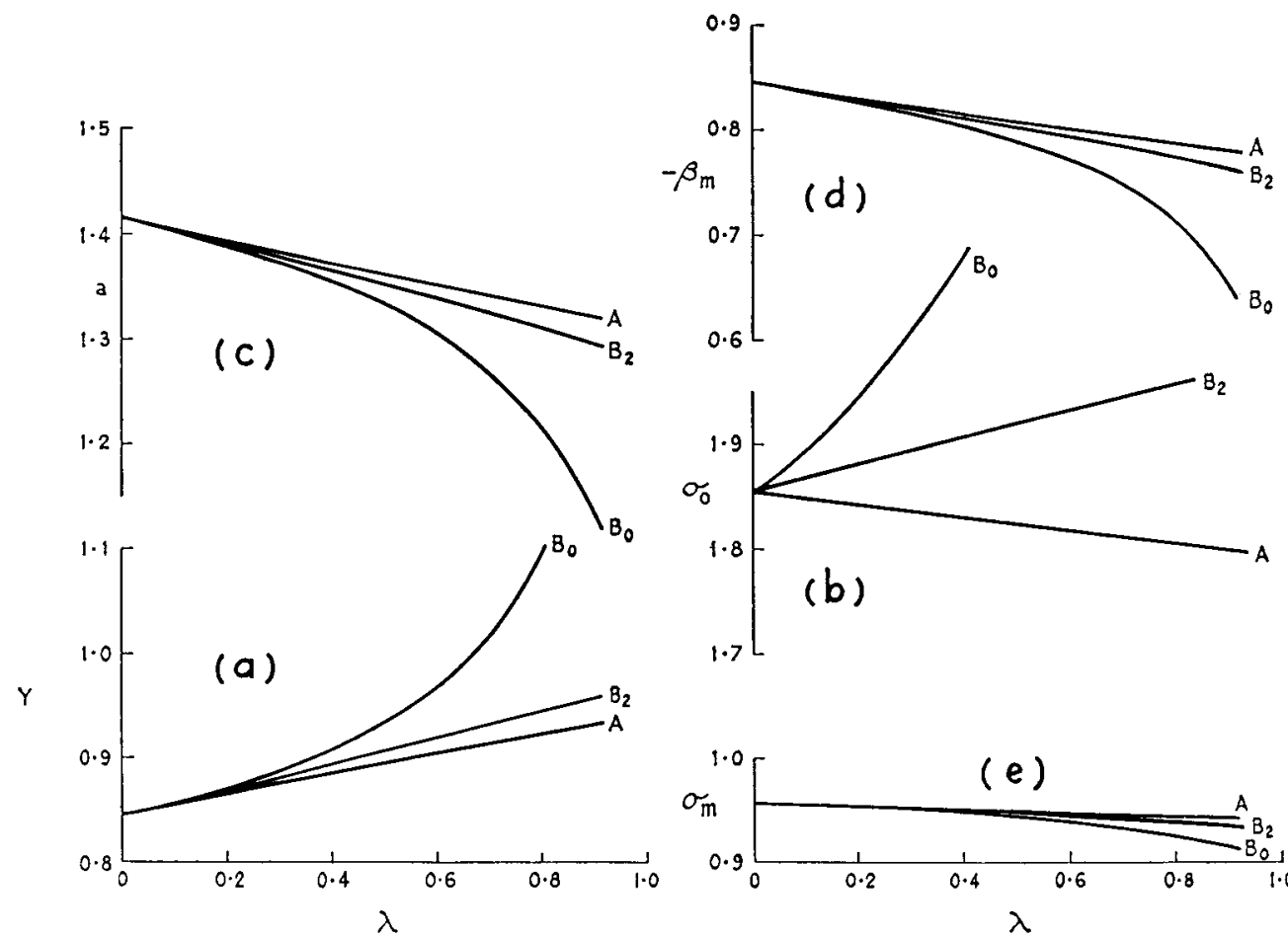

Fig. 5. The variation of some parameters of the loop with $\lambda$.

(a) $Y$, the half-height; (b) $\sigma_{0}$, the half-length; (c) $a$, the curvature of the axis; (d) $\beta_{m}$, the maximum value of $\sigma(d \theta / d \sigma)$; (e) $\sigma_{m}$, the value of $\sigma$ which maximises $\sigma(d \theta / d \sigma)$

A represents assumption A; $B_{0}$ represents assumption B with $\lambda_{R}=0 ; B_{2}$ represents assumption $\mathrm{B}$ with $\lambda_{R}=\frac{1}{2}(\lambda+1)$
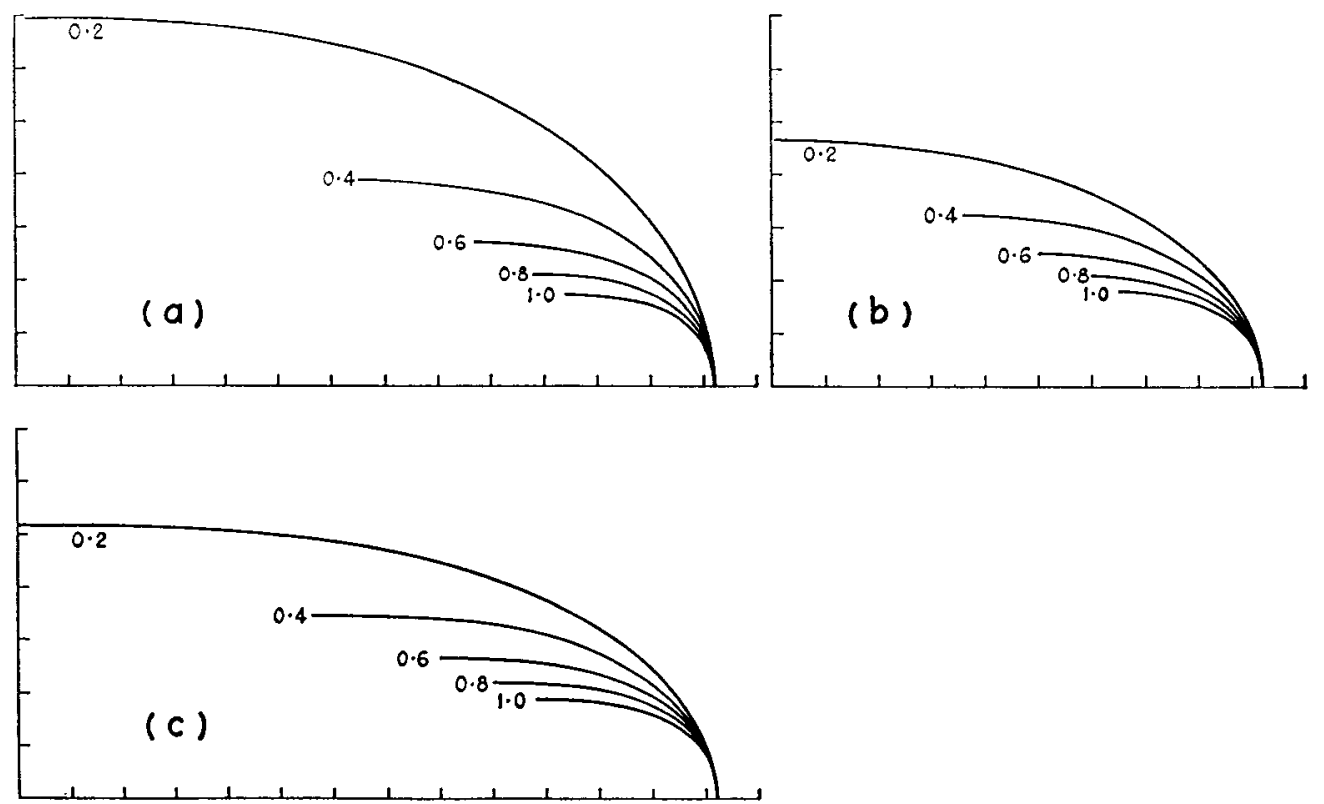

Fig. 6. The shapes of the bends as plotted by the computer

(a) Assumption A $\lambda=0.2$; (b) Assumption $\mathrm{A} \lambda=0.5$; (c) Assumption $\mathrm{B} \lambda=0.5 \lambda_{R}=0$

Figures on the curves represent $c^{*} / c=\left(P / P_{*}\right)^{\frac{1}{2}}$ 
(i) To calculate the height of the loop, $Y=\int_{0}^{\sigma_{0}} \sin \theta d \sigma$. The value of $Y$ was normalised so that

$$
Y\left(c_{*}\right)=1.0,
$$

and $\left(Y\left(c_{*} / c\right)\right)$ was plotted against $\left(c_{*} / c\right)$ by the computer's $X-Y$ recorder. This graph corresponds to $Y P^{\frac{1}{2}}-P^{\frac{1}{2}}$ (normalised), or

(ii) To plot the shape of the loop:

$$
X=\int_{0}^{\sigma} \cos \theta d \sigma ; Y=\int_{0}^{\sigma} \sin \theta d \sigma .
$$

\section{Results Obtained by Solution of the Equations}

\subsection{Bending}

Because of the assumption that $k_{R}$ is independent of $c$ in bending, the form of the equations is such that the shape of the loop, and hence the parameters of its dimensionless form, are independent of the load (i.e. of $c$ ).

The way in which the main parameters of the loop (half-height, $Y$, half-length, $\sigma_{0}$; curvature at axis, $a ; \beta_{m}$ and $\sigma_{m}$ ) vary with $\lambda$, under various postulates, is shown in Fig. 5. The actual shape of the loops, in a few cases are shown by the cases $\left(c_{*} / c\right)=1$ in Fig. 6 .

\subsection{Unbending}

The only parameter upon which the solution of the unbending equations depends is $\left(c_{*} / c\right)$ or $\left(P / P_{*}\right)^{\frac{1}{2}}$, and hence the form of the solution depends
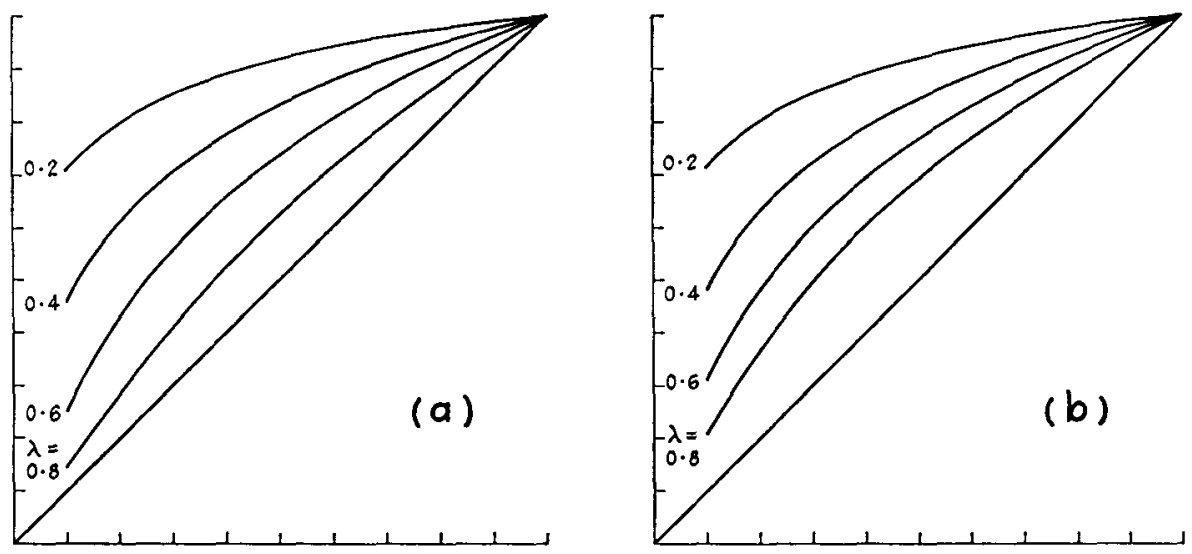

Fig. 7. Graphs of $Y\left(c_{*} / c\right) \sim c_{*} / c$ for values of $\lambda$ marked under (a) Assumption $A$ and, (b) Assumption B with $\lambda_{R}=0$. 
only on this ratio, and is independent of the maximum load applied. The shapes of the unbending curves in a few cases are shown in Fig. 6, and the resulting graphs of $Y\left(c_{*} / c\right) \sim\left(c_{*} / c\right)$ are shown in Fig. 7 .

\section{Discussion and Comparison with some Experimental Results on Cloth Loops}

The shape of $Y P^{\frac{1}{2}} \sim P^{\frac{1}{2}}$ curves for experimental loops of fabrics do not very closely approximate the $Y\left(c_{*} / c\right) \sim\left(c_{*} / c\right)$ curves predicted from the theory. Experimental curves, compared to some theoretical ones, are shown in Fig. 8. Down to a value of $\left(c_{*} / c\right)$ of about 0.4 (i.e. down to $P \cong 0.2 P_{*}$ ), a reasonable degree of agreement can be obtained by using a rather high value of $\lambda(0.4-0.6)$, which value is much higher than would be expected from the recovery angle experiments (2), (3).

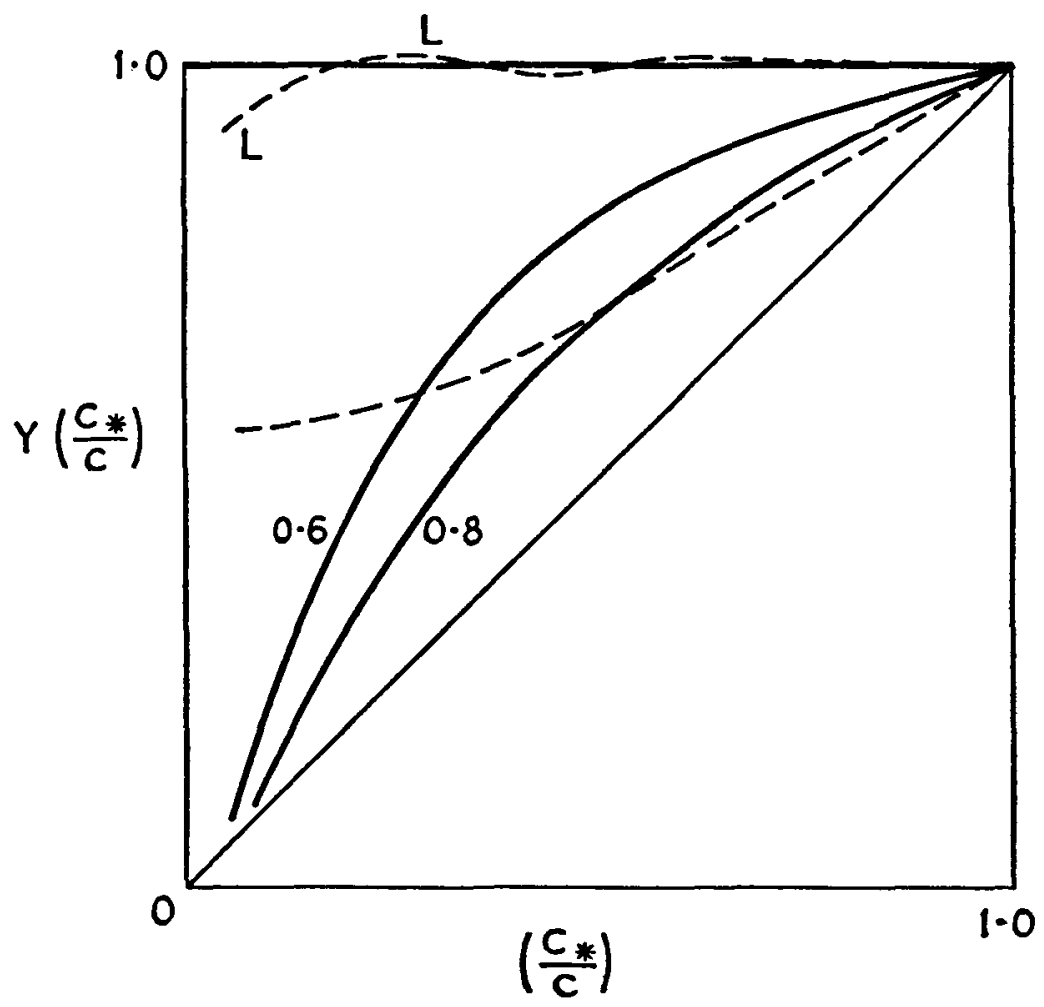

Fig. 8. Solid lines: Typical theoretical curves with $\lambda$ marked. $L$ indicates loading curves or $\lambda=0$.

Broken lines: Typical experimental curve. $L$ again indicates loading. 
It is possible that a worsted cloth does have a rather higher plasticity at high bending strains such as used in our tests than at low bending strains as used by Bostwick (2), (3). The upward swing of the experimental $Y P^{\frac{1}{2}}$ curves as $P^{\frac{1}{2}}$ decreases may be partly due to the fact that tension need not be zero at the end of the bend in the experimental case, as the loop has two bends which may sustain a tension in the fabric between them. Time varying effects, which have not yet been considered, may also be interfering.

\section{References}

[1] I. M. Stuart, P. Hetherington \& K. Baird, 'An Investigation of cloth bending Part I. Compression of looped fabrics', Textile Res. J. 36 (1966), 803-808.

[2] C. O. Bostwick, 'Analysing the wrinkling of textile materials by a new technique', Svenska Textil forskningsinstitute Report No. 3, Gothenburg, 1961.

[3] C. O. Bostwick, 'Comparison of some methods for testing wrinkle recovery'. Am. Dyestuff Reptr. 51, (1962), 386-393.

[4] N. E. Waters, 'Stored energy in flexure', Brit. J. Appl. Phys. 14 (1963), 908-911.

[5] D. G. Stechert, 'Simple flexibility tests', Anal. Chem. 25 (1953), 1730-1733.

[6] I. M. Stuart \& R. K. Mann, 'An Investigation of cloth bending Part II. The remanent curvature hypothesis', Text. Res. J. 37 (1967), 613-618.

Division of Textile Physics

CSIRO Wool Research Laboratories

Ryde, Sydney, Australia
Gordon Institute of Technology Fenwick St., Geelong, Australia 